\title{
Search for exotic particles at NA62
}

\author{
Riccardo Lollini*广 \\ Università degli Studi di Perugia \\ INFN - Sezione di Perugia \\ E-mail: riccardo.lollini@pg.infn.it
}

The NA62 experiment at the CERN SPS is currently taking data to measure the ultra-rare decay $K^{+} \rightarrow \pi^{+} v \bar{v}$. Owing to the high beam-energy and a hermetic detector coverage, NA62 also has the opportunity to directly search for a plaethora of long-lived beyond-the Standard Model particles, such as Heavy Neutral Leptons, Axion-like Particles and Dark Photons. We will review the status of this searches and give prospects for future data taking at NA62.

XXVI International Workshop on Deep-Inelastic Scattering and Related Subjects (DIS2018) 16-20 April 2018

Kobe, Japan

* Speaker.

${ }^{\dagger}$ On behalf of the NA62 Collaboration: R. Aliberti, F. Ambrosino, R. Ammendola, B. Angelucci, A. Antonelli, G. Anzivino, R. Arcidiacono, M. Barbanera, A. Biagioni, L. Bician, C. Biino, A. Bizzeti, T. Blazek, B. Bloch-Devaux, V. Bonaiuto, M. Boretto, M. Bragadireanu, D. Britton, F. Brizioli, M.B. Brunetti, D. Bryman, F. Bucci, T. Capussela, A. Ceccucci, P. Cenci, V. Cerny, C. Cerri, B. Checcucci, A. Conovaloff, P. Cooper, E. Cortina Gil, M. Corvino, F. Costantini, A. Cotta Ramusino, D. Coward, G. D’Agostini, J. Dainton, P. Dalpiaz, H. Danielsson, N. De Simone, D. Di Filippo, L. Di Lella, N. Doble, B. Dobrich, F. Duval, V. Duk, J. Engelfried, T. Enik, N. Estrada-Tristan, V. Falaleev, R. Fantechi, V. Fascianelli, L. Federici, S. Fedotov, A. Filippi, M. Fiorini, J. Fry, J. Fu, A. Fucci, L. Fulton, E. Gamberini, L. Gatignon, G. Georgiev, S. Ghinescu, A. Gianoli, M. Giorgi, S. Giudici, F. Gonnella, E. Goudzovski, C. Graham, R. Guida, E. Gushchin, F. Hahn, H. Heath, T. Husek, O. Hutanu, D. Hutchcroft, L. Iacobuzio, E. Iacopini, E. Imbergamo, B. Jenninger, K. Kampf, V. Kekelidze, S. Kholodenko, G. Khoriauli, A. Khotyantsev, A. Kleimenova, A. Korotkova, M. Koval, V. Kozhuharov, Z. Kucerova, Y. Kudenko, J. Kunze, V. Kurochka, V.Kurshetsov, G. Lanfranchi, G. Lamanna, G. Latino, P. Laycock, C. Lazzeroni, M. Lenti, G. Lehmann Miotto, E. Leonardi, P. Lichard, L. Litov, R. Lollini, D. Lomidze, A. Lonardo, P. Lubrano, M. Lupi, N. Lurkin, D. Madigozhin, I. Mannelli, G. Mannocchi, A. Mapelli, F. Marchetto, R. Marchevski, S. Martellotti, P. Massarotti, K. Massri, E. Maurice, M. Medvedeva, A. Mefodev, E. Menichetti, E. Migliore, E. Minucci, M. Mirra, M. Misheva, N. Molokanova, M. Moulson, S. Movchan, M. Napolitano, I. Neri, F. Newson, A. Norton, M. Noy, T. Numao, V. Obraztsov, A. Ostankov, S. Padolski, R. Page, V. Palladino, C. Parkinson, E. Pedreschi, M. Pepe, M. Perrin-Terrin, L. Peruzzo, P. Petrov, F. Petrucci, R. Piandani, M. Piccini, J. Pinzino, I. Polenkevich, L. Pontisso, Yu. Potrebenikov, D. Protopopescu, M. Raggi, A. Romano, P. Rubin, G. Ruggiero, V. Ryjov, A. Salamon, C. Santoni, G. Saracino, F. Sargeni, V. Semenov, A. Sergi, A. Shaikhiev, S. Shkarovskiy, D. Soldi, V. Sougonyaev, M. Sozzi, T. Spadaro, F. Spinella, A. Sturgess, J. Swallow, S. Trilov, P. Valente, B. Velghe, S. Venditti, P. Vicini, R. Volpe, M. Vormstein, H. Wahl, R. Wanke, B. Wrona, O. Yushchenko, M. Zamkovsky, A. Zinchenko. 


\section{The NA62 experiment}

NA62 is a fixed target experiment located at the North Area of the CERN SPS. The experiment aims at measuring the branching ratio of the very rare decay $K^{+} \rightarrow \pi^{+} v \bar{v}$ with a $10 \%$ precision in 3 years of data taking. In order to do this, a $400 \mathrm{GeV} / \mathrm{c}$ proton beam extracted from the SPS collides on a beryllium target, producing a $75 \mathrm{GeV} / \mathrm{c}$ positively charged hadron beam which contains a $6 \%$ $K^{+}$. The Standard Model prediction on the branching fraction:

$$
B R_{t h}\left(K^{+} \rightarrow \pi^{+} v \bar{v}\right)=(8.4 \pm 1.0) \times 10^{-11}[1]
$$

comes with a very small theoretical uncertainty, and a precise experimental value will be an important test for the unitarity of the CKM matrix. Moreover, a different value for the branching ratio is expected in a number of physics beyond the Standard Model scenarios (custodial Randall-Sundrum [2], MSSM analysis [3][4], simplified Z/Z' models [5], littlest Higgs with T-parity [6], LFU violation models [7]). The branching ratio for this process was already measured by E787 and E949 at BNL and it is consistent with the Standard Model, though the associated uncertainties are very large:

$$
B R_{\exp }\left(K^{+} \rightarrow \pi^{+} v \bar{v}\right)=17.3_{-10.5}^{+11.5} \times 10^{-11}[8] .
$$

In addition to this, NA62 has the potential to explore several new physics models, as described in section 2 .

\subsection{The NA62 detector}

In the upstream region a Cherenkov differential counter (KTAG) identifies the kaons in the beam, while a tracker (GigaTracker) tracks the particles in the beam. The GigaTracker consists of three stations of silicon trackers placed directly in the beam line. Right after the last station of the GigaTracker, a charged veto (CHANTI) rejects catastrophic events where particles in the beam interacted inelastically with the last station of the GigaTracker.

After the fiducial decay volume, in the downstream region, a magnetic spectrometer (STRAW) measures the momentum of the decay products. A RICH detector separates pions, muons and electrons, and a liquid Krypton calorimeter ( $\mathrm{LKr}$ ) provides a measurement of the energy. The muon veto system (MUV) consists of three different subdetector: the hadronic calorimeters MUV1 and MUV2, and the MUV3 detector, placed after a $80 \mathrm{~cm}$ thick iron wall, that acts as a fast muon veto at trigger level. The $\mathrm{LAV}, \mathrm{LKr}$, IRC and SAC detectors provide hermetic photon rejection, covering angles between zero and $50 \mathrm{mrad}$.

Finally, it is possible to run the experiment in dump-mode: two copper collimators (TAXes) placed $25 \mathrm{~m}$ after the Beryllium target and $1.6 \mathrm{~m}$ long can be closed, acting as a beam dump. In this case, only muons and neutrinos can reach the sub-detectors.

\section{Exotic searches at NA62}

Several new physics searches are carried out by the NA62 Collaboration in parallel with the $K^{+} \rightarrow \pi^{+} v \bar{v}$ measurement. These analyses include the search for heavy neutral leptons, axionlike particles, dark photons, lepton flavour and lepton number violations. Various features make 
NA62 particularly suitable for exotic searches: a high intensity setup, a flexible trigger system, high-frequency tracking of beam particles, redundant PID and ultra high-efficiency photon vetoes. In the following sections the main results regarding exotic searches in NA62 will be presented.

\subsection{Heavy Neutral Leptons}

The origin of neutrino masses remains an open question in particle physics. At the same time, many other observations are not explained in the context of the Standard Model, such as the baryon asymmetry in the universe and the origin of the cold dark matter. The vMSM (Neutrino Minimal Standard Model) is a particular extention of the SM that is consistent with all these observations. The model predicts three heavy sterile neutrinos $N_{i}$, weakly coupled to classical neutrinos [9]. The first heavy neutrino, $N_{1}$, should have a mass of $\sim O(10 \mathrm{keV})$ and it would be the ideal candidate for dark matter. The other two heavy neutrinos, $N_{2}$ and $N_{3}$, should have a larger mass of about $\sim O(1 \mathrm{GeV})$ and they would bring an extra $\mathrm{CP}$ violation that would make them responsible for the baryon asymmetry and the tiny SM neutrino masses via the seesaw mechanism.

In the NA62 detector, heavy neutral leptons can be produced in meson decays like $K^{+} \rightarrow l^{+} N$, where $l^{+}=\left(e^{+}, \mu^{+}\right)$. The branching ratio for the process is given by:

$$
B R\left(K^{+} \rightarrow l^{+} N\right)=B R\left(K^{+} \rightarrow l^{+} v\right) \cdot \rho_{l}\left(m_{N}\right) \cdot\left|U_{l 4}\right|^{2},
$$

where $\rho_{l}\left(m_{N}\right)$ is a kinematic factor and $\left|U_{l 4}\right|^{2}$ is the mixing to SM neutrinos.

During 2015, NA62 collected $\sim 3 \times 10^{8} \mathrm{~K}^{+} \rightarrow e^{+} v$ and $\sim 1 \times 10^{8} \mathrm{~K}^{+} \rightarrow \mu^{+} v$ decays. The results are shown in fig. 1. Since no signal events have been observed, NA62 put upper limits on the couplings of $\mathrm{HNL}$ in the mass range $[170,448] \mathrm{MeV} / \mathrm{c}^{2}$ for $\left|U_{e 4}\right|^{2}$ and $[250,373] \mathrm{MeV} / \mathrm{c}^{2}$ for $\left|U_{\mu 4}\right|^{2}$ [10]. The analysis of 2016-2018 data is ongoing and could put much stronger limits on the HNL masses and couplings.
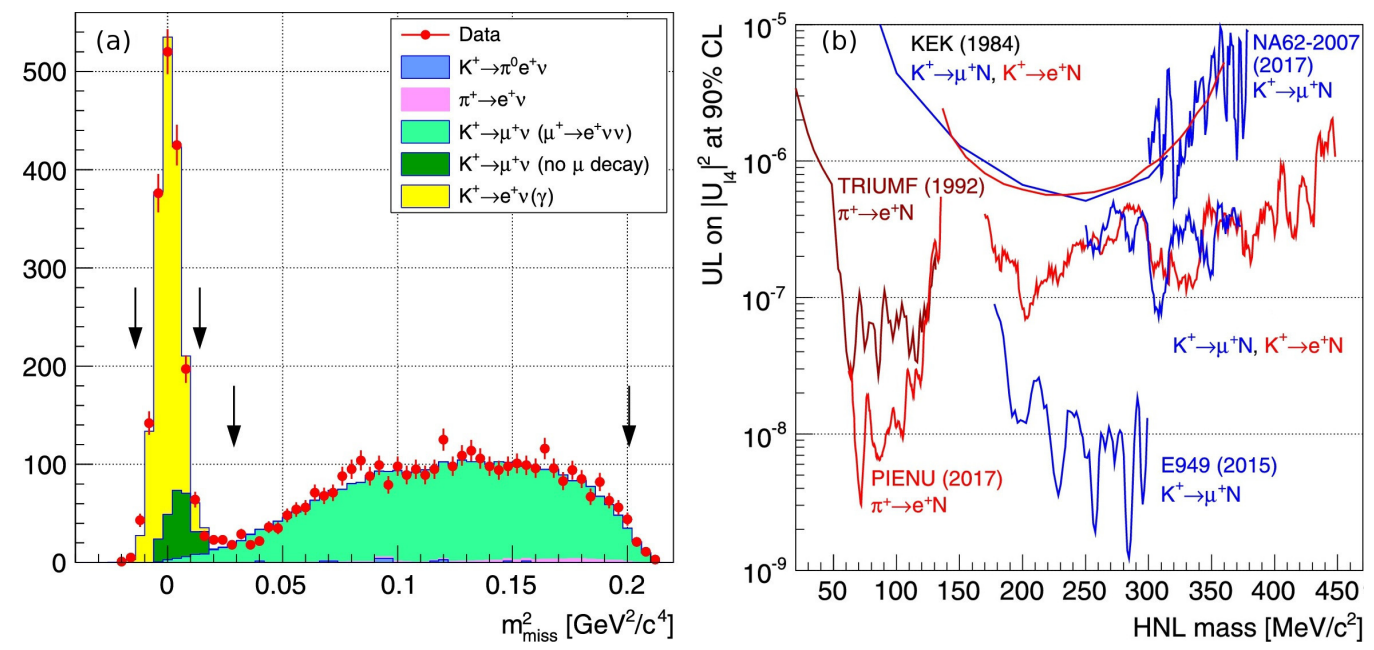

Figure 1: (a) Distribution of the $m_{\text {miss }}^{2}$ variable for data and simulated events passing the $e^{+}$selection. Pairs of vertical lines indicate the boundaries of the SM and HNL signal regions. (b) Upper limits at 90\% CL on $\left|U_{l 4}\right|^{2}$ obtained for each assumed HNL mass compared to the limits established by earlier HNL production searches. 


\subsection{Axion-Like Particles}

With its high beam energy and intensity, and the presence of ultra high efficiency photon vetoes, NA62 has the potential to discover new weakly coupled particles in the $\mathrm{MeV}$ to $\mathrm{GeV}$ mass range, such as the axion-like particle (ALP), a pseudoscalar coupled to two photons. For a high energy proton beam, it is possible to reliably compute the cross section for the production of ALPs in the $\mathrm{MeV}$ to $\mathrm{GeV}$ range via photon-photon fusion, the so-called Primakoff production[11]. In order to do this, NA62 has to be run in dump-mode: two copper collimator (the so-called TAXes), placed $25 \mathrm{~m}$ after the target, each $1.6 \mathrm{~m}$ long, can be closed and hence act as a beam dump. This is also important to suppress the background, since all the particles in the beam will be stopped in the dump, except for muons and neutrinos. The Primakoff production is particularly interesting for proton beam dump experiments for two main reasons. First of all, the cross section of the process is proportional to $\sim Z^{2}$, thus ALPs production is strongly enhanced for high-Z beam dumps. Second, ALPs produced via Primakoff production have typically a very small transverse momentum, therefore even small detectors far from the production point will have a good acceptance. Thus Primakoff production is both the cleanest and the dominant production mode. The NA62 detector would be particularly suitable for ALPs searches. Since the experiment aims at measuring the $K^{+} \rightarrow \pi^{+} v \bar{v}$ decay, a very efficient photon veto is mandatory: this opens up the possibility of looking for the decay of a ALPs into two photons $(A L P \rightarrow \gamma \gamma)$. Unfortunately the beam dump operation mode is incompatible with the $K^{+} \rightarrow \pi^{+} v \bar{v}$ measurement. On the other hand, as shown in fig. 2, a single day of data taking in dump mode would make NA62 competitive with other experiments looking for ALPs[11].
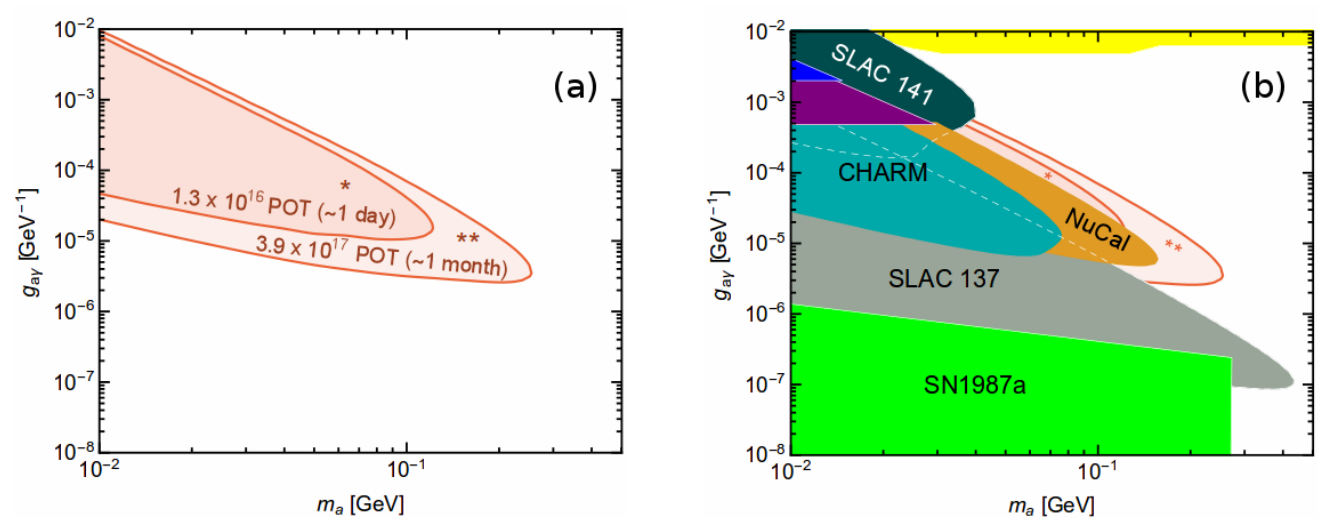

Figure 2: (a) Expected sensitivity of NA62 in the $\left(m_{a}, g_{a \gamma}\right)$ plane for a number of protons on target roughly equivalent to one day and one month of data taking. $m_{a}$ and $g_{a \gamma}$ are, respectively, the ALP mass and the ALPphoton coupling. (b) The same plot, where the NA62 projected sensitivity is compared with the constraints from other experiments.

\subsection{Dark Photons}

In several hidden sectors models, an extra $U(1)$ gauge simmetry leads to an invisible vector boson $A^{\prime}$, also known as dark photon, which acts as a portal between the SM and the dark sector[12]. These dark photons would also provide a possible explanation for the positron excess in cosmic 
rays and the muon (g-2) measurement[13]. Dark photon are coupled to SM photons with a small coupling $\varepsilon$ :

$$
\mathscr{L}=\frac{\varepsilon}{2} F_{\mu v} F^{\prime \mu v} .
$$

NA62 has potential to search both for the visible and the invisible decay of the dark photon. In the following, the results for the invisible decay of the dark photon will be presented.

\subsubsection{Dark Photon invisible decay at NA62}

Because of the small coupling of the dark photon to the classical photon, if this exotic particle exists the following decay chain could be observed:

$$
K^{+} \rightarrow \pi^{+} \pi^{0}, \pi^{0} \rightarrow \gamma A^{\prime}, \quad A^{\prime} \rightarrow \text { invisible }
$$

where the $A^{\prime}$ is not detected. The main kinematic variable used for this search is the squared missing mass, defined as:

$$
M_{\text {miss }}^{2}=\left(P_{K}-P_{\pi}-P_{\gamma}\right)^{2},
$$

where $P_{K}, P_{\pi}$ and $P_{\gamma}$ are, respectively, the 4-momenta of the kaon, the charged pion and the photon. In the squared missing mass distribution, a peak is expected around $M_{A^{\prime}}^{2}$. The main background comes from the SM decay $\pi^{0} \rightarrow \gamma \gamma$ when a photon is lost: in this case, $M_{m i s s}^{2}$ peaks around zero. Since the width of the background peak is purely due to resolution effects, which are basically simmetrical, the background can be evaluated directly from the data, analysing the negative tail in the $M_{m i s s}^{2}$ distribution.

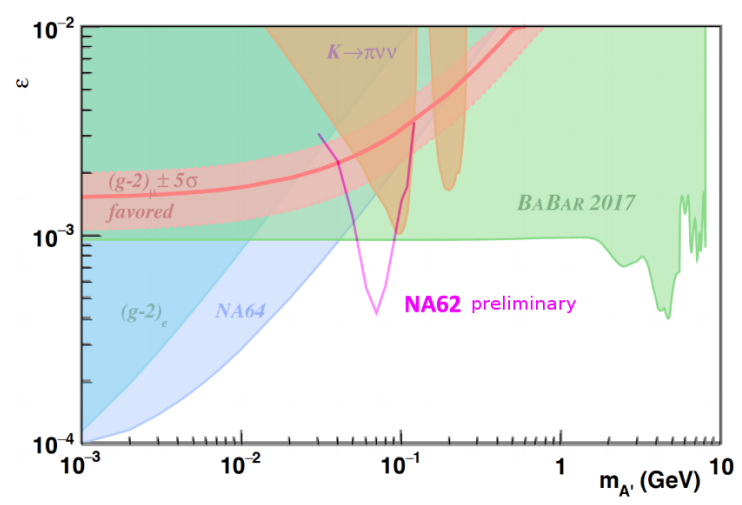

Figure 3: $90 \% \mathrm{CL}$ exclusion plot in the $\left(\varepsilon, M_{A^{\prime}}\right)$ plane for $\pi^{0} \rightarrow \gamma A^{\prime}$ events with an undetected $A^{\prime}$. The limits from E949[14], NA64[15] and BaBar[16] are shown together with the NA62 preliminary result obtained with the $5 \%$ of 2016 data.

In NA62, this kind of search was performed using $1.5 \times 10^{10} \mathrm{~K}^{+}$decays, corresponding to about the 5\% of data collected in 2016. The results are shown in fig. 3: no statistically significant excess was observed and upper limits were computed in the $\left(\varepsilon, M_{A^{\prime}}\right)$ plane[17].

\section{Conclusions}

NA62 is currently taking data to measure the FCNC decay $K^{+} \rightarrow \pi^{+} v \bar{v}$ at the CERN SPS and the analysis of 2016-2018 data will improve significantly the state of the art. At the same time, 
several hidden-sector searches can be performed with NA62, such as searches for heavy neutral leptons, axion-like particles and dark photons, besides lepton flavour and lepton number violating processes. Some of these searches, such as the search for ALPs, require a beam-dump data taking, which is not compatible with the normal data taking. A Run3 for NA62 would offer the opportunity to have some dedicated runs for these kind of searches.

\section{References}

[1] A. Buras et al., $K^{+} \rightarrow \pi^{+} v \bar{v}$ and $K_{L} \rightarrow \pi^{0} v \bar{v}$ in the Standard Model: status and perspectives, JHEP 11 (2015) 033.

[2] M. Blanke et al., Rare K and B Decays in a Warped Extra Dimension with Custodial Protection, JHEP 0903 (2009) 108.

[3] T. Blažek et al., Left-left squark mixing, $K+\rightarrow \pi^{+} v \bar{v}$ and minimal supersymmetry with large tan $\beta$, Int.J.Mod.Phys. A29 (2014) no.27.

[4] G. Isidori et al., Exploring the flavour structure of the MSSM with rare K decays, JHEP 0608 (2006) 064.

[5] A. Buras et al., $K \rightarrow \pi v \bar{v}$ and $\varepsilon^{\prime} / \varepsilon$ in simplified new physics models, JHEP 11 (2015) 166.

[6] M. Blanke et al., Quark flavour observables in the Littlest Higgs model with T-parity after LHC Run 1, Eur.Phys.J. C76 (2016) no.4, 182.

[7] G. Isidori et al., Probing Lepton Flavour Universality with $K \rightarrow \pi v \bar{v}$ decays, Eur.Phys.J. C77 (2017) no. 9,618 .

[8] A. V. Artamonov et al. (E949 Collaboration), Study of the decay $K^{+} \rightarrow \pi^{+} v \bar{v}$ in the momentum region $140<P_{\pi}<199 \mathrm{MeV} / c$, Phys.Rev.D 79, 092004 (2009).

[9] T. Asaka et al., The vMSM, dark matter and baryon asymmetry of the universe, Phys Lett B 620 (2005) 17

[10] NA62 Collaboration, Search for heavy neutral lepton production in $K^{+}$decays, Phys. Lett. B778 (2018) 137-145.

[11] B. Döbrich et al., ALPtraum: ALP production in proton beam dump experiments, JHEP 1602 (2016) 018.

[12] B. Holdom, Two U(1)'s and $\varepsilon$ charge shifts, Phys. Lett. B166 (1986) 196.

[13] M. Pospelov, Secluded U(1) below the weak scale, Phys. Rev. D80 (2009) 095002.

[14] E949 Collaboration (A. V. Artamonov et al.), Study of the decay $K^{+} \rightarrow \pi^{+} v \bar{v}$ in the momentum region $140<P_{\pi}<199 \mathrm{MeV} / c$, Phys. Rev. D79 (2009) 092004.

[15] NA64 Collaboration (D. Banerjee et al.), Search for Invisible Decays of Sub-GeV Dark Photons in Missing-Energy Events at the CERN SPS, Phys. Rev. Lett. 118 (2017) no.1, 011802.

[16] BaBar Collaboration (J. P. Lees et al.), Search for Invisible Decays of a Dark Photon Produced in $e^{+} e^{-}$Collisions at BaBar, Phys. Rev. Lett. 119 (2017) no.13, 131804.

[17] M. Corvino, Search for $K^{+} \rightarrow \pi^{+} v \bar{v}$ and Future Searches for Exotic Processes at NA62, Int. J. Mod. Phys. Conf. Ser., 46, 1860068 (2018). 